Mathematical Modelling and Analysis

Volume 11 Number 2, 2006, PAGes 173-186

(c) 2006 Technika ISSN 1392-6292 print, ISSN 1648-3510 online

\title{
INTEGRAL TRANSFORM WITH THE EXTENDED GENERALIZED MITTAG-LEFFLER FUNCTION
}

\section{A.A. KILBAS and A.A. KOROLEVA}

\section{Belarusian State University}

Faculty of Mathematics and Mechanics, Belarusian State University, 220050 Minsk, Belarus

E-mail: kilbas@bsu.by; akoroleva@tut.by

Received February 15, 2006; revised March 25, 2006; published online May 25, 2006

Abstract. The paper is devoted to the study of the integral transform

$$
\left(\mathcal{E}(\alpha, \beta)_{n} f\right)(x)=\int_{0}^{\infty} \mathcal{E}\left((\alpha, \beta)_{n} ;-x t\right) f(t) d t \quad(x>0)
$$

containing the special function $\mathcal{E}\left((\alpha, \beta)_{n} ; z\right)$ generalizing the Mittag-Leffler type function in the space $\mathcal{L}_{\nu, r}(1 \leq r \leq \infty, \nu \in \mathbf{R})$ of Lebesgue measurable functions on $\mathbf{R}_{+}=(0,+\infty)$ such that $\|f\|_{\nu, r}<\infty$, where

$$
\|f\|_{\nu, r} \equiv\left(\int_{0}^{\infty}\left|t^{\nu} f(t)\right| \frac{d t}{t}\right)^{1 / r}<\infty \quad(1 \leq r<\infty) ;\|f\|_{\nu, \infty} \equiv \operatorname{esssup}_{t>0}\left|t^{\nu} f(t)\right| .
$$

Mapping properties such as the boundedness, the range, the representation and the inversion of the considered transform are proved. The results are based on the representation of the considered transform as the $H$-transform.

Key words: Mittag-Leffler type function, spaces of $p$-summable functions, $H$ function, $\mathbf{H}$-transform

\section{Introduction}

Our paper is devoted to the study of the integral transform of the form

$$
\left.\left(\mathcal{E}(\alpha, \beta)_{n} f\right)(x)=\int_{0}^{\infty} \mathcal{E}(\alpha, \beta)_{n} ;-x t\right) f(t) d t \quad(x>0),
$$


involving the special function

$$
\mathcal{E}\left((\alpha, \beta)_{n} ; z\right)=\frac{1}{2 \pi i} \int_{\mathcal{L}} \frac{\Gamma(s) \Gamma(1-s)}{\prod_{i=1}^{n} \Gamma\left(\beta_{i}-\alpha_{i} s\right)}(-z)^{-s} d s \quad(z \neq 0)
$$

with real $\alpha_{i}, \beta_{i} \in \mathbf{R}(i=1, \ldots, n)$ in the kernel. Here $\mathcal{L}$ is the one of the following specially chosen infinite contours, which separate all poles of Gammafunction $\Gamma(s)$ to the left and all poles of Gamma-function $\Gamma(1-s)$ to the right:

(a) $\mathcal{L}=\mathcal{L}_{-\infty}$ is a left loop located in a horizontal strip starting at the point $-\infty+i \varphi_{1}$ and terminating at the point $-\infty+i \varphi_{2}, \quad-\infty<\varphi_{1}<\varphi_{2}<\infty$.

(b) $\mathcal{L}=\mathcal{L}_{+\infty}$ is a right loop located in the horizontal strip starting at the point $+\infty+i \varphi_{1}$ and terminating at the point $+\infty+i \varphi_{2}, \quad-\infty<\varphi_{1}<$ $\varphi_{2}<\infty$.

The function (1.2) with complex $\alpha_{i}, \beta_{i} \in \mathbf{C}(i=1, \ldots, n)$ was introduced in [7], where the following conditions for its existence were proved:

$$
\begin{aligned}
& \mathcal{L}=\mathcal{L}_{-\infty}, \quad \Re\left(\alpha_{1}+\ldots+\alpha_{n}\right)>0 ; \\
& \mathcal{L}=\mathcal{L}_{+\infty}, \quad \Re\left(\alpha_{1}+\ldots+\alpha_{n}\right)<0 .
\end{aligned}
$$

The following series representations of (1.2) were also established in [7]:

$$
\mathcal{E}\left((\alpha, \beta)_{n} ; z\right)=\sum_{k=0}^{\infty} c_{k} z^{k} \equiv E\left((\alpha, \beta)_{n} ; z\right), \quad c_{k}=\frac{1}{\prod_{i=1}^{n} \Gamma\left(\alpha_{i} k+\beta_{i}\right)}
$$

for $\mathcal{L}=\mathcal{L}_{-\infty}$ and $\sum_{i=1}^{n} \Re\left(\alpha_{i}\right)>0$, while

$$
\mathcal{E}\left((\alpha, \beta)_{n} ; z\right)=\sum_{k=0}^{\infty} \frac{d_{k}}{z^{k+1}}, \quad d_{k}=\frac{1}{\prod_{i=1}^{n} \Gamma\left(-\alpha_{i} k-\alpha_{i}+\beta_{i}\right)},
$$

for $\mathcal{L}=\mathcal{L}_{+\infty}$ and $\sum_{i=1}^{n} \Re\left(\alpha_{i}\right)<0$.

The function $E\left((\alpha, \beta)_{n} ; z\right)$ in the form (1.5) as the generalized MittagLeffler function was introduced by Hadid and Luchko [6]. Therefore we call (1.2) the extended generalized Mittag-Leffler function. This function is the generalization of the classical Mittag-Leffler function $E_{\alpha, \beta}(z)[2,3,5]$ :

$$
E_{\alpha, \beta}(z)=\sum_{k=0}^{\infty} \frac{z^{k}}{\Gamma(\alpha k+\beta)}(\alpha>0 ; \beta \in \mathbf{R} ; z \in \mathbf{C}),
$$

and more general function $E_{\alpha_{1}, \beta_{1} ; \alpha_{2}, \beta_{2}}(z)[1]$ :

$E_{\alpha_{1}, \beta_{1} ; \alpha_{2}, \beta_{2}}(z)=\sum_{k=0}^{\infty} \frac{z^{k}}{\Gamma\left(\alpha_{1} k+\beta_{1}\right) \Gamma\left(\alpha_{2} k+\beta_{2}\right)} ;\left(\alpha_{1}, \alpha_{2}>0, \beta_{1}, \beta_{2} \in \mathbf{R} ; z \in \mathbf{C}\right)$. 
Let's note, that some special Bessel type functions $[4,8]$ are expressed in terms of $E_{\alpha_{1}, \beta_{1} ; \alpha_{2}, \beta_{2}}(z)$, i.e. the Bessel function of the first kind

$$
J_{\nu}(z)=\left(\frac{z}{2}\right)^{\nu} E_{1, \nu+1 ; 1,1}\left(-\frac{z^{2}}{4}\right),
$$

the Struve function

$$
H_{\nu}(z)=\left(\frac{z}{2}\right)^{\nu+1} E_{1, \nu+3 / 2 ; 1,3 / 2}\left(-\frac{z^{2}}{4}\right),
$$

the Lommel function

$$
S_{\mu, \nu}(z)=\frac{z^{\mu+1}}{4} \Gamma\left(\frac{\mu-\nu+1}{2}\right) \Gamma\left(\frac{\mu+\nu+1}{2}\right) E_{1, \frac{\mu-\nu+1}{2} ; 1, \frac{\mu+\nu+1}{2}}\left(-\frac{z^{2}}{4}\right),
$$

the Bessel-Maitland function

$$
J_{\nu}^{\mu}(z)=E_{\mu, \nu+1 ; 1,1}(-z),
$$

the generalized Bessel-Maitland function

$$
J_{\nu, \lambda}^{\mu}(z)=\left(\frac{z}{2}\right)^{\nu+2 \lambda} E_{\mu, \nu+\lambda+1 ; 1, \lambda+1}\left(-\frac{z^{2}}{4}\right) .
$$

Recently interest to the investigation of the functions

$$
E_{\alpha, \beta}(z), \quad E_{\alpha_{1}, \beta_{1} ; \alpha_{2}, \beta_{2}}(z), \quad E\left((\alpha, \beta)_{n} ; z\right)
$$

and the integral transforms with such functions in the kernels has considerably increased due to their close connections with the theory of fractional integration and to the solutions of so called integral and differential equations of fractional order; see the survey paper [9] in this connection.

In our paper we study the mapping properties such as the boundedness, the range, the representation and the invertability of the transform (1.1) on the space $\mathcal{L}_{\nu, r}(1 \leq r \leq \infty, \nu \in \mathbf{R})$ of Lebesgue measurable functions on $\mathbf{R}_{+}=(0,+\infty)$ such that $\|f\|_{\nu, r}<\infty$, where

$$
\|f\|_{\nu, r} \equiv\left(\int_{0}^{\infty}\left|t^{\nu} f(t)\right| \frac{d t}{t}<\infty\right)^{1 / r} ;\|f\|_{\nu, \infty} \equiv \operatorname{esssup}_{t>0}\left|t^{\nu} f(t)\right| .
$$

Our investigations are based on the representation of this transform in the form of more general integral transform

$$
(\mathbf{H} f)(x)=\int_{0}^{\infty} H_{p, q}^{m, n}\left[x t \mid \begin{array}{l}
\left(a_{i}, \alpha_{i}\right)_{1, p} \\
\left(b_{j}, \beta_{j}\right)_{1, q}
\end{array}\right] f(t) d t,
$$

with the so-called $H$-function in the kernel. Such a $H_{p, q}^{m, n}(z)$-function is defined for integers $m, n, p, q(0 \leq m \leq q, 0 \leq n \leq p)$, for complex $a_{i}, b_{j}$ and positive $\alpha_{i}, \beta_{j}(1 \leq i \leq p ; 1 \leq j \leq q)$ by the following equation: 


$$
H_{p, q}^{m, n}(z)=H_{p, q}^{m, n}\left[z \mid \begin{array}{l}
\left(a_{i}, \alpha_{i}\right)_{1, p} \\
\left(b_{j}, \beta_{j}\right)_{1, q}
\end{array}\right]=\frac{1}{2 \pi i} \int_{\mathcal{L}} \mathcal{H}_{p, q}^{m, n}(s) z^{-s} d s
$$

with

$$
\mathcal{H}_{p, q}^{m, n}(s):=\frac{\prod_{j=1}^{m} \Gamma\left(b_{j}+\beta_{j} s\right) \prod_{l=1}^{n} \Gamma\left(1-a_{l}-\alpha_{l} s\right)}{\prod_{l=n+1}^{r} \Gamma\left(a_{l}+\alpha_{l} s\right) \prod_{j=m+1}^{q} \Gamma\left(1-b_{j}-\beta_{j} s\right)} .
$$

Here $\mathcal{L}$ is a specially chosen infinite contour $\left(\mathcal{L}=\mathcal{L}_{-\infty}, \mathcal{L}=\mathcal{L}_{+\infty}\right.$, etc. $)$, and an empty product, if it occurs, being taken to be one. One may find the theory of this function in the books by Mathai and Saxena [10], Srivastava, Gupta and Goyal [13], Prudnikov, Brychkov and Marichev [11, Section 8.3] and Kilbas and Saigo [8, Chapters 1 and 2].

The paper is organized as follows. Section 2 deals with the representation of $\mathcal{E}(\alpha, \beta)_{n} f$ as special cases of the $H$-transform (1.8). Sections $3-4$ are devoted to the $\mathcal{L}_{\nu, r}$-theory of the transform (1.1) in the case when $1<p<n, \alpha_{i}>$ $0(i=1, \ldots, p)$ and $\alpha_{i}<0(i=p+1, \ldots, n)$. The inversion relations are given in Section 5.

\section{2. $\mathcal{E}(\alpha, \beta)_{n}$-Transform as the $\boldsymbol{H}$-Transform}

By (1.9)-(1.10), the extended generalized Mittag-Leffler function $\mathcal{E}\left((\alpha, \beta)_{n} ; z\right)$ is represented in the form of the $H$-function, and such a representation is different in dependence on values of real parameters $\alpha_{i}(i=1, \ldots, n)$ :

i) If $\alpha_{i}>0(i=1, \ldots, n)$ and $\mathcal{L}=\mathcal{L}_{-\infty}$, then

$$
\mathcal{E}\left((\alpha, \beta)_{n} ; z\right)=H_{1, n+1}^{1,1}\left[z \mid \begin{array}{l}
(0,1) \\
(0,1),\left(1-\beta_{1}, \alpha_{1}\right), \ldots,\left(1-\beta_{n}, \alpha_{n}\right)
\end{array}\right] .
$$

ii) If $\alpha_{i}>0(i=1, \ldots, p, p<n), \alpha_{i}<0(i=p+1, \ldots, n)$ and either

$$
\begin{aligned}
& \sum_{i=1}^{n} \alpha_{i}>0, \mathcal{L}=\mathcal{L}_{-\infty}, \text { or } \sum_{i=1}^{n} \alpha_{i}<0, \mathcal{L}=\mathcal{L}_{+\infty}, \text { then } \\
& \mathcal{E}\left((\alpha, \beta)_{n} ; z\right)=H_{n-p+1, p+1}^{1,1}\left[\begin{array}{l}
\left.z \mid \begin{array}{l}
(0,1),\left(\beta_{p+1},-\alpha_{p+1}\right), \ldots,\left(\beta_{n},-\alpha_{n}\right) \\
(0,1),\left(1-\beta_{1}, \alpha_{1}\right), \ldots,\left(1-\beta_{p}, \alpha_{p}\right)
\end{array}\right] .
\end{array}\right.
\end{aligned}
$$

iii) If $\alpha_{i}<0(i=1, \ldots, p, p<n)$ and $\alpha_{i}>0(i=p+1, \ldots, n)$ and either

$$
\begin{aligned}
& \sum_{i=1}^{n} \alpha_{i}>0, \mathcal{L}=\mathcal{L}_{-\infty}, \text { or } \sum_{i=1}^{n} \alpha_{i}<0, \mathcal{L}=\mathcal{L}_{+\infty}, \text { then } \\
& \mathcal{E}\left((\alpha, \beta)_{n} ; z\right)=H_{p+1, n-p+1}^{1,1}\left[\begin{array}{l}
\left.z \mid \begin{array}{l}
(0,1),\left(\beta_{1},-\alpha_{1}\right), \ldots,\left(\beta_{p},-\alpha_{p}\right) \\
(0,1),\left(1-\beta_{p+1}, \alpha_{p+1}\right), \ldots,\left(1-\beta_{n}, \alpha_{n}\right)
\end{array}\right] .
\end{array}\right.
\end{aligned}
$$


iv) If $\alpha_{i}<0(i=1, \ldots, n)$ and $\mathcal{L}=\mathcal{L}_{+\infty}$, then

$$
\mathcal{E}\left((\alpha, \beta)_{n} ; z\right)=H_{n+1,1}^{1,1}\left[z \mid \begin{array}{l}
(0,1),\left(\beta_{1},-\alpha_{1}\right), \ldots,\left(\beta_{n},-\alpha_{n}\right) \\
(0,1)
\end{array}\right] .
$$

By (2.1)-(2.4), the transform $\mathcal{E}(\alpha, \beta)_{n} f$ in (1.1) has the following representations:

A) If $\alpha_{i}>0(i=1, \ldots, n)$

$$
\mathcal{E}\left((\alpha, \beta)_{n} ; z\right)=H_{1, n+1}^{1,1}\left[z \mid \begin{array}{l}
(0,1) \\
(0,1),\left(1-\beta_{1}, \alpha_{1}\right), \ldots,\left(1-\beta_{n}, \alpha_{n}\right)
\end{array}\right] .
$$

B) If $\alpha_{i}>0(i=1, \ldots, p, p<n), \alpha_{i}<0(i=p+1, \ldots, n)$ and either $\sum_{i=1}^{n} \alpha_{i}>0, \mathcal{L}=\mathcal{L}_{-\infty}$, or $\sum_{i=1}^{n} \alpha_{i}<0, \mathcal{L}=\mathcal{L}_{+\infty}$, then

$$
\begin{gathered}
\left(\mathcal{E}(\alpha, \beta)_{n} f\right)(x)= \\
\int_{0}^{\infty} H_{n-p+1, p+1}^{1,1}\left[x t \mid \begin{array}{l}
(0,1),\left(\beta_{p+1},-\alpha_{p+1}\right), \ldots,\left(\beta_{n},-\alpha_{n}\right) \\
(0,1),\left(1-\beta_{1}, \alpha_{1}\right), \ldots,\left(1-\beta_{p}, \alpha_{p}\right)
\end{array}\right] f(t) d t .
\end{gathered}
$$

C) If $\alpha_{i}<0(i=1, \ldots, p, p<n)$ and $\alpha_{i}>0(i=p+1, \ldots, n)$ and either $\sum_{i=1}^{n} \alpha_{i}>0, \mathcal{L}=\mathcal{L}_{-\infty}$, or $\sum_{i=1}^{n} \alpha_{i}<0, \mathcal{L}=\mathcal{L}_{+\infty}$, then

$$
\begin{gathered}
\left(\mathcal{E}(\alpha, \beta)_{n} f\right)(x)= \\
\int_{0}^{\infty} H_{p+1, n-p+1}^{1,1}\left[x t \mid \begin{array}{l}
(0,1),\left(\beta_{1},-\alpha_{1}\right), \ldots,\left(\beta_{p},-\alpha_{p}\right) \\
(0,1),\left(1-\beta_{p+1}, \alpha_{p+1}\right), \ldots,\left(1-\beta_{n}, \alpha_{n}\right)
\end{array}\right] f(t) d t .
\end{gathered}
$$

D) If $\alpha_{i}<0,(i=1, \ldots, n)$ and $\mathcal{L}=\mathcal{L}_{+\infty}$, then

$$
\mathcal{E}\left((\alpha, \beta)_{n} f\right)(x)=\int_{0}^{\infty} H_{n+1,1}^{1,1}\left[x t \mid \begin{array}{l}
(0,1),\left(\beta_{1},-\alpha_{1}\right), \ldots,\left(\beta_{n},-\alpha_{n}\right) \\
(0,1)
\end{array}\right] f(t) d t .
$$

The properties of the $H$-function (1.9) and of the $\boldsymbol{H}$-transform (1.8) depend on the following numbers $a^{*}, \Delta, \mu, \delta, a_{1}^{*}, a_{2}^{*}, \alpha, \beta, \alpha_{0}, \beta_{0}$ :

$$
\begin{aligned}
& a^{*}=\sum_{i=1}^{n} \alpha_{i}-\sum_{i=n+1}^{p} \alpha_{i}+\sum_{j=1}^{m} \beta_{j}-\sum_{j=m+1}^{q} \beta_{j}, \quad \Delta=\sum_{j=1}^{q} \beta_{j}-\sum_{i=1}^{p} \alpha_{i}, \\
& \mu=\sum_{j=1}^{q} b_{j}-\sum_{i=1}^{p} a_{i}+\frac{p-q}{2}, \quad \delta=\prod_{i=1}^{p} \alpha_{i}^{-\alpha_{i}} \prod_{j=1}^{q} \beta_{j}^{\beta_{j}} \\
& a_{1}^{*}=\sum_{j=1}^{m} \beta_{j}-\sum_{i=n+1}^{p} \alpha_{i}, \quad a_{2}^{*}=\sum_{i=1}^{n} \alpha_{i}-\sum_{i=m+1}^{q} \beta_{j},
\end{aligned}
$$




$$
\begin{aligned}
& \alpha= \begin{cases}-\min _{1 \leq j \leq m}\left[\frac{\Re\left(b_{j}\right)}{\beta_{j}}\right], & \text { if } m>0, \\
-\infty, & \text { if } m=0,\end{cases} \\
& \beta= \begin{cases}\min _{1 \leq i \leq n}\left[\frac{1-\operatorname{Re}\left(a_{i}\right)}{\alpha_{i}}\right], & \text { if } n>0, \\
\infty, & \text { if } n=0,\end{cases} \\
& \alpha_{0}= \begin{cases}\max _{m+1 \leq j \leq q}\left[\frac{\operatorname{Re}\left(b_{j}\right)-1}{\beta_{j}}+1\right], & \text { if } q>m \\
-\infty, & \text { if } q=m,\end{cases} \\
& \beta_{0}= \begin{cases}\min _{n+1 \leq i \leq p}\left[\frac{\operatorname{Re}\left(a_{i}\right)}{\alpha_{i}}+1\right], & \text { if } p>n, \\
\infty, & \text { if } p=n .\end{cases}
\end{aligned}
$$

The mapping properties, such as the boundedness, the range and the representation of the $\mathbf{H}$-transform on the $\mathcal{L}_{\nu, r}$-spaces with any $\nu \in \mathbf{R}$ and $1 \leq r \leq \infty$ are different in the following nine cases:

(1) $a^{*}=\Delta=\operatorname{Re}(\mu)=0$;

(2) $a^{*}=\Delta=0, \operatorname{Re}(\mu)<0 ;(3) a^{*}=0, \Delta>0$;

(4) $a^{*}=0, \Delta<0$;

(5) $a_{1}^{*}>0, a_{2}^{*}>0$;

(6) $a_{1}^{*}>0, a_{2}^{*}=0$

(7) $a_{1}^{*}=0, a_{2}^{*}>0$;

(8) $a^{*}>0, a_{1}^{*}>0, a_{2}^{*}<0$;

(9) $a^{*}>0, a_{1}^{*}<0, a_{2}^{*}>0$

while the invertibility of the $\boldsymbol{H}$-transform is valid for $a^{*}=0$.

The corresponding results, giving $\mathcal{L}_{\nu, 2^{-}}$and $\mathcal{L}_{\nu, r}$-theory of the $\mathbf{H}$-transform, are established in the above book by Kilbas and Saigo [7, Theorems 3.6-3.7] and [7, Theorems 4.1-4.10], respectively, while the invertibility of such a transform is presented in [7, Theorems 4.11-4.14]. We only note that the range $\mathbf{H}\left(\mathcal{L}_{\nu, r}\right)$ of the $\mathbf{H}$-transform (1.8) is characterized in terms of the following transforms:

Erdelyi-Kober type fractional integration operators $I_{0+; \sigma, \eta}^{\alpha} f$ and $I_{-; \sigma, \eta}^{\alpha} f$ :

$$
\begin{gathered}
\left(I_{0+; \sigma, \eta}^{\alpha} f\right)(x)=\frac{\sigma x^{-\sigma(\alpha+\eta)}}{\Gamma(\alpha)} \int_{0}^{x}\left(x^{\sigma}-t^{\sigma}\right)^{\alpha-1} t^{\sigma \eta+\sigma-1} f(t) d t \quad(x>0), \\
\left(I_{-; \sigma, \eta}^{\alpha} f\right)(x)=\frac{\sigma x^{\sigma \eta}}{\Gamma(\alpha)} \int_{x}^{\infty}\left(t^{\sigma}-x^{\sigma}\right)^{\alpha-1} t^{\sigma(1-\alpha-\eta)-1} f(t) d t \quad(x>0),
\end{gathered}
$$

defined for $\alpha \in \mathbf{C}(\Re(\alpha)>0), \sigma>0$ and $\eta \in \mathbf{C}$; and the modified Laplace transform $L_{k, \alpha} f$ :

$$
\left(L_{k, \alpha} f\right)(x)=\int_{0}^{\infty}(x t)^{-\alpha} e^{-|k|(x t)^{1 / k}} f(t) d t \quad(x>0),
$$

with $k \in \mathbf{R}(k \neq 0)$ and $\alpha \in \mathbf{C}$; and the modified Hankel transform $H_{k, \eta} f$ : 


$$
\left(H_{k, \eta} f\right)(x)=\int_{0}^{\infty}(x t)^{1 / k-1 / 2} J_{\eta}\left(|k|(x t)^{1 / k}\right) f(t) d t \quad(x>0),
$$

with $k \in \mathbf{R}(k \neq 0)$ and $\eta \in \mathbf{C}(\Re(\eta)>-3 / 2)$; and the elementary transform

$$
\left(M_{\zeta} f\right)(x)=x^{\zeta} f(x) \quad(\zeta \in \mathbf{C}) .
$$

Note that when $\sigma=1,(2.17)$ and (2.18) coincide with the so-called Erdelyi-Kober operators [12, Section 18.1]:

$$
\begin{gathered}
\left(I_{\eta, \alpha}^{+} f\right)(x) \equiv\left(I_{0+; 1, \eta}^{\alpha} f\right)(x)=\frac{x^{-\alpha-\eta}}{\Gamma(\alpha)} \int_{0}^{x}(x-t)^{\alpha-1} t^{\eta} f(t) d t \quad(x>0), \\
\left(K_{\eta, \alpha}^{-} f\right)(x) \equiv\left(I_{-; 1, \eta}^{\alpha} f\right)(x)=\frac{x^{\eta}}{\Gamma(\alpha)} \int_{x}^{\infty}(t-x)^{\alpha-1} t^{-\alpha-\eta} f(t) d t \quad(x>0),
\end{gathered}
$$

for $k=1$ and $\alpha=0(2.20)$ yields the classical Laplace transform

$$
(L f)(x) \equiv\left(L_{1,0} f\right)(x)=\int_{0}^{\infty} e^{-x t} f(t) d t \quad(x>0) .
$$

We shall use for $1 \leq r<\infty$ the notation $r^{\prime}$ and $\gamma(r)$ as follows:

$$
\frac{1}{r}+\frac{1}{r^{\prime}}=1, \quad \gamma(r)=\max \left[\frac{1}{r}, \frac{1}{r^{\prime}}\right] .
$$

\section{3. $\mathcal{L}_{\nu, r^{-}}$Theory of $\mathcal{E}(\alpha, \beta)_{n}$-Transform when $\alpha_{i}>0$} $(i=1, \ldots, p, p<n)$ and $\alpha_{i}<0(i=p+1, \ldots, n)$

Here we present $\mathcal{L}_{\nu, r}$-theory of the $\mathcal{E}(\alpha, \beta)_{n}$-transform (1.1) in the case $1<$ $p<n, \alpha_{i}>0(i=1, \ldots, p)$ and $\alpha_{i}<0(i=p+1, \ldots, n)$. By (2.2) and (1.9), the constants (2.9)-(2.15) take the following forms:

$$
\begin{aligned}
& a^{*}=2+\sum_{i=p+1}^{n} \alpha_{i}-\sum_{i=1}^{p} \alpha_{i} ; \Delta=\sum_{i=1}^{n} \alpha_{i} ; \mu=n / 2-\sum_{i=1}^{n} \beta_{i} ; \\
& a_{1}^{*}=1+\sum_{i=p+1}^{n} \alpha_{i} ; a_{2}^{*}=1-\sum_{i=1}^{p} \alpha_{i} ; \alpha=0 ; \beta=1 ; \\
& \alpha_{0}=1-\min _{1 \leq i \leq p} \frac{\Re\left(\beta_{i}\right)}{\alpha_{i}}, \beta_{0}=1-\max _{p+1 \leq i \leq n} \frac{\Re\left(\beta_{i}\right)}{\alpha_{i}},
\end{aligned}
$$

By this calculation, all nine cases (1)-(9) in (2.16) are possible. In this section we consider cases (1)-(4) in (2.16) when $a^{*}=0$.

From [8, Theorems 4.1 and 4.2] we deduce the first two results when $\alpha_{p+1}+\ldots+\alpha_{n}=-1$ and $\alpha_{1}+\ldots+\alpha_{p}=1$. 
Theorem 1. Let $p<n, \alpha_{i}>0(i=1, \ldots, p), \alpha_{i}<0(i=p+1, \ldots, n)$, $\beta_{i} \in \mathbf{C}(i=1, \ldots, n)$ be such that $\alpha_{p+1}+\ldots+\alpha_{n}=-1, \alpha_{1}+\ldots+\alpha_{p}=1$ and $\Re\left(\beta_{1}+\ldots+\beta_{n}\right)=n / 2$, and let $0<\nu<1,1<r<\infty$.

(a) The transform $\mathcal{E}(\alpha, \beta)_{n}$ defined on $\mathcal{L}_{\nu, 2}$ can be extended to $\mathcal{L}_{\nu, r}$ as an element of $\left[\mathcal{L}_{\nu, r}, \mathcal{L}_{1-\nu, r}\right]$.

(b) If $1<r \leq 2$, then the transform $\mathcal{E}(\alpha, \beta)_{n}$ is one-to-one on $\mathcal{L}_{\nu, r}$, and there holds the equality

$$
\left(\mathcal{M E}(\alpha, \beta)_{n} f\right)(s)=\frac{\Gamma(s) \Gamma(1-s)}{\prod_{i=1}^{n} \Gamma\left(\beta_{i}-\alpha_{i} s\right)}(\mathcal{M} f)(1-s) \quad(\Re(s)=1-\nu) .
$$

(c) If there holds the condition

$$
s \neq \frac{\beta_{1}+k}{\alpha_{1}}, \ldots, s \neq \frac{\beta_{n}+l}{\alpha_{n}}(k, l=0,1,2, \cdots) \text { for } \Re(s)=1-\nu,
$$

then the transform $\mathcal{E}(\alpha, \beta)_{n}$ is one-to-one from $\mathcal{L}_{\nu, r}$ onto $\mathcal{L}_{1-\nu, r}$ :

$$
\mathcal{E}(\alpha, \beta)_{n}\left(\mathcal{L}_{\nu, r}\right)=\mathcal{L}_{1-\nu, r}
$$

(d) If $f \in \mathcal{L}_{\nu, r}$ and $g \in \mathcal{L}_{\nu, r^{\prime}}$, then there holds the relation

$$
\int_{0}^{\infty} f(x)\left(\mathcal{E}(\alpha, \beta)_{n} g\right)(x) d x=\int_{0}^{\infty}\left(\mathcal{E}(\alpha, \beta)_{n} f\right)(x) g(x) d x
$$

(e) If $f \in \mathcal{L}_{\nu, r}, \lambda \in \mathbf{C}$ and $h>0$, then $\mathcal{E}(\alpha, \beta)_{n} f$ is given by

$$
\begin{aligned}
& \left(\mathcal{E}(\alpha, \beta)_{n} f\right)(x)=h x^{1-(\lambda+1) / h} \frac{d}{d x} x^{(\lambda+1) / h} \int_{0}^{\infty} H_{n-p+2, p+2}^{1,2} \\
& \times\left[x t \mid \begin{array}{c}
(-\lambda, h),(0,1),\left(\beta_{p+1},-\alpha_{p+1}\right), \ldots,\left(\beta_{n},-\alpha_{n}\right) \\
(0,1),\left(1-\beta_{1}, \alpha_{1}\right), \ldots,\left(1-\beta_{p}, \alpha_{p}\right),(-\lambda-1, h)
\end{array}\right] f(t) d t
\end{aligned}
$$

for $\operatorname{Re}(\lambda)>(1-\nu) h-1$, while

$$
\begin{aligned}
& \left(\mathcal{E}(\alpha, \beta)_{n} f\right)(x)=-h x^{1-(\lambda+1) / h} \frac{d}{d x} x^{(\lambda+1) / h} \int_{0}^{\infty} H_{n-p+2, p+2}^{2,1} \\
& \quad \times\left[x t \mid \begin{array}{c}
(0,1),\left(\beta_{p+1},-\alpha_{p+1}\right), \ldots,\left(\beta_{n},-\alpha_{n}\right),(-\lambda, h) \\
(-\lambda-1, h),(0,1),\left(1-\beta_{1}, \alpha_{1}\right), \ldots,\left(1-\beta_{p}, \alpha_{p}\right)
\end{array}\right] f(t) d t
\end{aligned}
$$

for $\operatorname{Re}(\lambda)<(1-\nu) h-1$. 
Theorem 2. Let $p<n, \alpha_{i}>0(i=1, \ldots, p), \alpha_{i}<0(i=p+1, \ldots, n)$, $\beta_{i} \in \mathbf{C}(i=1, \ldots, n)$ be such that $\alpha_{p+1}+\ldots+\alpha_{n}=-1, \alpha_{1}+\ldots+\alpha_{p}=1$ and $\Re\left(\beta_{1}+\ldots+\beta_{n}\right)>n / 2$, and let $0<\nu<1,1<r<\infty$.

(a) The transform $\mathcal{E}(\alpha, \beta)_{n}$ defined on $\mathcal{L}_{\nu, 2}$ can be extended to $\mathcal{L}_{\nu, r}$ as an element of $\left[\mathcal{L}_{\nu, r}, \mathcal{L}_{1-\nu, r}\right]$ for all $s \geq r$ such that $1 / s>1 / r+n / 2-\Re\left(\beta_{1}+\right.$ $\left.\ldots+\beta_{n}\right)$.

(b) If $1<r \leq 2$, then the transform $\mathcal{E}(\alpha, \beta)_{n}$ is one-to-one on $\mathcal{L}_{\nu, r}$, and there holds the equality (3.4).

(c) Let $k>0$. If the condition (3.5) is satisfied, then $\mathcal{E}(\alpha, \beta)_{n}$ is one-to-one on $\mathcal{L}_{\nu, r}$ and there holds

$$
\mathcal{E}(\alpha, \beta)_{n}\left(\mathcal{L}_{\nu, r}\right)=I_{-; k, 0}^{\beta_{1}+\ldots+\beta_{n}-n / 2}\left(\mathcal{L}_{1-\nu, r}\right)=I_{0+; k,(1 / k)-1}^{\beta_{1}+\ldots+\beta_{n}-n / 2}\left(\mathcal{L}_{1-\nu, r}\right)
$$

If the condition (3.5) is not satisfied, then $\mathcal{E}(\alpha, \beta)_{n}\left(\mathcal{L}_{\nu, r}\right)$ is a subset of $I_{-; k, 0}^{\beta_{1}+\ldots+\beta_{n}-n / 2}\left(\mathcal{L}_{1-\nu, r}\right)$ and $I_{0+; k,(1 / k)-1}^{\beta_{1}+\ldots+\beta_{n}-n / 2}\left(\mathcal{L}_{1-\nu, r}\right)$.

(d) If $f \in \mathcal{L}_{\nu, r}$ and $g \in \mathcal{L}_{\nu, s}$ with $1<r<\infty, 1<s<\infty$ and $1 \leq 1 / r+1 / s<$ $\Re\left(\beta_{1}+\ldots+\beta_{n}\right)+1-n / 2$, then the relation (3.7) holds.

(e) If $f \in \mathcal{L}_{\nu, r}, \lambda \in \mathbf{C}$ and $h>0$, then $\mathcal{E}(\alpha, \beta)_{n} f$ is given by in (3.8) for $\Re(\lambda)>(1-\nu) h-1$, while in (3.9) for $\Re(\lambda)<(1-\nu) h-1$. Furthermore, $\mathcal{E}(\alpha, \beta)_{n}$ is given by (1.9) and (2.6).

The next result follows from [8, Theorems 4.3 and 4.4].

Theorem 3. Let $p<n, \alpha_{i}>0(i=1, \ldots, p)$ and $\alpha_{i}<0(i=p+1, \ldots, n)$ be such that $2-\alpha_{1}-\ldots-\alpha_{p}+\alpha_{p+1}+\ldots+\alpha_{n}=0$ and $\alpha_{1}+\ldots+\alpha_{n} \neq 0$, and let $0<\nu<1,1<r<\infty$, and let $\beta_{i} \in \mathbf{C}(i=1, \ldots, n)$ be such that $\Re\left(\beta_{1}+\ldots+\beta_{n}\right)-\left(\alpha_{1}+\ldots+\alpha_{n}\right)(1-\nu) \geq \gamma(r)+(n-1) / 2$.

(a) The transform $\mathcal{E}(\alpha, \beta)_{n}$ defined on $\mathcal{L}_{\nu, 2}$ can be extended to $\mathcal{L}_{\nu, r}$ as an element of $\left[\mathcal{L}_{\nu, r}, \mathcal{L}_{1-\nu, s}\right]$ for all $s$ with $r \leq s<\infty$ such that $s^{\prime} \geq\left(\Re\left(\beta_{1}+\ldots+\beta_{n}\right)-\left(\alpha_{1}+\ldots+\alpha_{n}\right)(1-\nu)+(1-n) / 2\right)^{-1}$ with $\frac{1}{s}+$ $\frac{1}{s^{\prime}}=1$.

(b) If $1<r \leq 2$, then the transform $\mathcal{E}(\alpha, \beta)_{n}$ is one-to-one on $\mathcal{L}_{\nu, r}$, and there holds the equality (3.4).

(c) Let $\Delta=\alpha_{1}+\ldots+\alpha_{n}, \mu=n / 2-\beta_{1}-\ldots-\beta_{n}$, and $\eta=\beta_{1}+\ldots+\beta_{n}-n / 2-1$, $\Re(\eta)>-1$. If the condition (3.5) is satisfied, then the transform $\mathcal{E}(\alpha, \beta)_{n}$ is one-to-one on $\mathcal{L}_{\nu, r}$, and

$$
\mathcal{E}(\alpha, \beta)_{n}\left(\mathcal{L}_{\nu, r}\right)=\left(M_{\mu / \Delta+1 / 2} H_{\Delta, \eta}\right)\left(\mathcal{L}_{\nu-1 / 2+\Re(\mu) / \Delta, r}\right)
$$

When the condition in (3.5) is not satisfied, then $\mathcal{E}(\alpha, \beta)_{n}\left(\mathcal{L}_{\nu, r}\right)$ is a subset of the right-hand side of (3.10).

(d) If $f \in \mathcal{L}_{\nu, r}$ and $g \in \mathcal{L}_{\nu, s}, 1<s<\infty, 1 / s+1 / r \geq 1$ and $\operatorname{Re}\left(\beta_{1}+\ldots+\right.$ $\left.\beta_{n}\right)-\left(\alpha_{1}+\ldots+\alpha_{n}\right)(1-\nu) \geq \max [\gamma(r), \gamma(s)]+(n-1) / 2$, then the relation (3.7) holds. 
(e) If $f \in \mathcal{L}_{\nu, r}, \lambda \in \mathbf{C}, h>0$ and $\operatorname{Re}\left(\beta_{1}+\ldots+\beta_{n}\right)-\left(\alpha_{1}+\ldots+\alpha_{n}\right)(1-\nu) \geq$ $\gamma(r)+(n-1) / 2$, then $\mathcal{E}(\alpha, \beta)_{n} f$ is given by equation (3.8) for $\Re(\lambda)>$ $(1-\nu) h-1$, while by (3.9) for $\Re(\lambda)<(1-\nu) h-1$. If $\Re\left(\beta_{1}+\ldots+\beta_{n}\right)-$ $\left(\alpha_{1}+\ldots+\alpha_{n}\right)(1-\nu)>n / 2$, then $\mathcal{E}(\alpha, \beta)_{n} f$ is given by (1.9) and $(2.6)$.

\section{4. $\mathcal{L}_{\nu, r}$-Theory of $\mathcal{E}(\alpha, \beta)_{n}$-Transform when $\alpha_{i}>0$} $(i=1, \ldots, p, p<n)$ and $\alpha_{i}<0(i=p+1, \ldots, n)$. Continuation.

Here we present $\mathcal{L}_{\nu, r}$ theory of $\mathcal{E}(\alpha, \beta)_{n}$-transform in cases (5)-(9) in (2.16), when $a^{*}>0$. Next statement, following from [8, Theorem 4.5], presents the $\mathcal{L}_{\nu, r}$-theory of the transform $\mathcal{E}(\alpha, \beta)_{n}$.

Theorem 4. Let $p<n, \alpha_{i}>0(i=1, \ldots, p)$ and $\alpha_{i}<0(i=p+1, \ldots, n)$ be such that $2-\alpha_{1}-\ldots-\alpha_{p}+\alpha_{p+1}+\ldots+\alpha_{n}>0$, and let $0<\nu<1$, $1 \leq r \leq s<\infty$.

(a) The transform $\mathcal{E}(\alpha, \beta)_{n}$ defined on $\mathcal{L}_{\nu, 2}$ can be extended to $\mathcal{L}_{\nu, r}$ as an element of $\left[\mathcal{L}_{\nu, r}, \mathcal{L}_{1-\nu, s}\right]$. When $1 \leq r \leq 2$, then $\mathcal{E}(\alpha, \beta)_{n}$ is a one-to-one transform from $\mathcal{L}_{\nu, r}$ onto $\mathcal{L}_{1-\nu, s}$.

(b) If $f \in \mathcal{L}_{\nu, r}$ and $g \in \mathcal{L}_{\nu, s^{\prime}}$ with $1 / s+1 / s^{\prime}=1$, then the relation (3.7) holds.

Further five statements, following from [8, Theorems 4.6-4.9], characterize the boundedness and the range of the $\mathcal{E}(\alpha, \beta)_{n}$-transform in the above cases (5)-(9), respectively.

Theorem 5. Let $p<n, \alpha_{i}>0(i=1, \ldots, p)$ and $\alpha_{i}<0(i=p+1, \ldots, n)$ be such that $a_{1}^{*}=1+\sum_{i=p+1}^{n} \alpha_{i}>0$ and $a_{2}^{*}=1-\sum_{i=1}^{p} \alpha_{i}>0$, and let $0<\nu<1$, $1<r<\infty$, and let $\omega=n / 2+\alpha_{1}+\ldots+\alpha_{p}-\beta_{1}-\ldots-\beta_{n}$.

(a) If the condition in (3.5) is satisfied, or if $1<r \leq 2$ then the transform $\mathcal{E}(\alpha, \beta)_{n}$ is one-to-one on $\mathcal{L}_{\nu, r}$.

(b) If $\Re(\omega) \geq 0$ and the condition in (3.5) is valid, then

$$
\mathcal{E}(\alpha, \beta)_{n}\left(\mathcal{L}_{\nu, r}\right)=\left(L_{a_{1}^{*}, 0} L_{a_{2}^{*},-\omega / a_{2}^{*}}\right)\left(\mathcal{L}_{1-\nu, r}\right) .
$$

When the condition in (3.5) is not valid, $\mathcal{E}(\alpha, \beta)_{n}\left(\mathcal{L}_{\nu, r}\right)$ is the subset of the right-hand side of (4.1).

(c) If $\Re(\omega)<0$ and the condition in (3.5) is satisfied, then

$$
\mathcal{E}(\alpha, \beta)_{n}\left(\mathcal{L}_{\nu, r}\right)=\left(I_{-;, 1 / a_{1}^{*}, 0}^{-\omega} L_{a_{1}^{*}, 0} L_{a_{2}^{*}, 0}\right)\left(\mathcal{L}_{1-\nu, r}\right) .
$$

When the condition in (3.5) is not valid, $\mathcal{E}(\alpha, \beta)_{n}\left(\mathcal{L}_{\nu, r}\right)$ is the subset of the right-hand side of (4.2). 
Theorem 6. Let $p<n, \alpha_{i}>0(i=1, \ldots, p)$ and $\alpha_{i}<0(i=p+1, \ldots, n)$ be such that $a_{1}^{*}=1+\sum_{i=p+1}^{n} \alpha_{i}>0$ and $\sum_{i=1}^{p} \alpha_{i}=1$, and let $0<\nu<1,1<r<\infty$, and let $\omega=(n+1) / 2-\beta_{1}-\ldots-\beta_{n}$.

(a) If the condition in (3.5) is satisfied, or if $1<r \leq 2$ then the transform $\mathcal{E}(\alpha, \beta)_{n}$ is one-to-one on $\mathcal{L}_{\nu, r}$.

(b) If $\Re(\omega) \geq 0$ and the condition in (3.5) is valid, then

$$
\mathcal{E}(\alpha, \beta)_{n}\left(\mathcal{L}_{\nu, r}\right)=\left(L_{a_{1}^{*},-\omega / a_{1}^{*}}\right)\left(\mathcal{L}_{\nu, r}\right) .
$$

When the condition in (3.5) is not valid, $\mathcal{E}(\alpha, \beta)_{n}\left(\mathcal{L}_{\nu, r}\right)$ is the subset of the right-hand side of (4.3).

(c) If $\Re(\omega)<0$ and the condition in (3.5) is satisfied, then

$$
\mathcal{E}(\alpha, \beta)_{n}\left(\mathcal{L}_{\nu, r}\right)=\left(I_{-;, 1 / a_{1}^{*}, 0}^{-\omega} L_{a_{1}^{*}, 0}\right)\left(\mathcal{L}_{\nu, r}\right) .
$$

When the condition in (3.5) is not valid, $\mathcal{E}(\alpha, \beta)_{n}\left(\mathcal{L}_{\nu, r}\right)$ is the subset of the right-hand side of (4.3).

Theorem 7. Let $p<n, \alpha_{i}>0(i=1, \ldots, p)$ and $\alpha_{i}<0(i=p+1, \ldots, n)$ be such that $\sum_{i=p+1}^{n} \alpha_{i}=-1$ and $a_{2}^{*}=1-\sum_{i=1}^{p} \alpha_{i}>0$, and let $0<\nu<1$, $1<r<\infty$, and let $\omega=(n-1) / 2-\beta_{1}-\ldots-\beta_{n}+\alpha_{1}+\ldots+\alpha_{p}$.

(a) If the condition in (3.5) is satisfied, or if $1<r \leq 2$ then the transform $\mathcal{E}(\alpha, \beta)_{n}$ is one-to-one on $\mathcal{L}_{\nu, r}$.

(b) If $\Re(\omega) \geq 0$ and the condition in (3.5) is valid, then

$$
\mathcal{E}(\alpha, \beta)_{n}\left(\mathcal{L}_{\nu, r}\right)=\left(L_{-a_{2}^{*}, 1+\omega / a_{2}^{*}}\right)\left(\mathcal{L}_{\nu, r}\right) .
$$

When the condition in $(3.5)$ is not valid, $\mathcal{E}(\alpha, \beta)_{n}\left(\mathcal{L}_{\nu, r}\right)$ is the subset of the right-hand side of (4.5).

(c) If $\Re(\omega)<0$ and the condition in (3.5) is satisfied, then

$$
\mathcal{E}(\alpha, \beta)_{n}\left(\mathcal{L}_{\nu, r}\right)=\left(I_{0+;, 1 / a_{2}^{*},-\alpha_{1}-\ldots-\alpha_{p}}^{-\omega} L_{a_{2}^{*}, 1}\right)\left(\mathcal{L}_{\nu, r}\right) .
$$

When the condition in (3.5) is not valid, $\mathcal{E}(\alpha, \beta)_{n}\left(\mathcal{L}_{\nu, r}\right)$ is the subset of the right-hand side of (4.6).

Theorem 8. Let $p<n, \alpha_{i}>0(i=1, \ldots, p)$ and $\alpha_{i}<0(i=p+1, \ldots, n)$ be such that $a^{*}=2-\alpha_{1}-\ldots-\alpha_{p}+\alpha_{p+1}+\ldots+\alpha_{n}>0, a_{1}^{*}=1+\sum_{i=p+1}^{n} \alpha_{i}>0$ and $a_{2}^{*}=1-\sum_{i=1}^{p} \alpha_{i}<0$, and let $0<\nu<1,1<r<\infty$.

(a) If the condition in (3.5) is satisfied, or if $1<r \leq 2$ then the transform $\mathcal{E}(\alpha, \beta)_{n}$ is one-to-one on $\mathcal{L}_{\nu, r}$. 
(b) If the condition in (3.5) is valid, and let $\omega, \eta, \zeta \in \mathbf{C}$ be such that

$$
\begin{aligned}
& \omega=a^{*} \eta+\beta_{1}+\ldots+\beta_{n}-\frac{n+1}{2} ; \\
& a^{*} \Re(\eta) \geq \gamma(r)+2 a_{2}^{*}(\nu-1)+n / 2-\Re\left(\beta_{1}+\ldots+\beta_{n}\right) ; \\
& \Re(\eta)>\nu-1 ; \Re(\zeta)<1-\nu ;
\end{aligned}
$$

where $\gamma(r)$ is given in (2.25), then

$$
\begin{array}{r}
\mathcal{E}(\alpha, \beta)_{n}\left(\mathcal{L}_{\nu, r}\right)=\left(M_{\frac{1}{2}+\frac{\omega}{2 a_{2}^{*}}} \mathbb{H}_{-2 a_{2}^{*}, 2 a_{2}^{*} \zeta+\omega-1} \mathbb{L}_{\left.-a^{*}, 1 / 2+\eta-\frac{\omega}{2 a_{2}^{*}}\right)}\right. \\
\left(\mathcal{L}_{\frac{3}{2}-\nu+\frac{\Re(\omega)}{2 a_{2}^{*}}}, r\right) .
\end{array}
$$

When the condition in $(3.5)$ is not valid, $\mathcal{E}(\alpha, \beta)_{n}\left(\mathcal{L}_{\nu, r}\right)$ is the subset of the right-hand side of (4.7).

Theorem 9. Let $p<n, \alpha_{i}>0(i=1, \ldots, p)$ and $\alpha_{i}<0(i=p+1, \ldots, n)$ be such that $a^{*}=2-\alpha_{1}-\ldots-\alpha_{p}+\alpha_{p+1}+\ldots+\alpha_{n}>0, a_{1}^{*}=1+\sum_{i=p+1}^{n} \alpha_{i}<0$ and $a_{2}^{*}=1-\sum_{i=1}^{p} \alpha_{i}>0$, and let $0<\nu<1,1<r<\infty$.

(a) If the condition in (3.5) is satisfied, or if $1<r \leq 2$ then the transform $\mathcal{E}(\alpha, \beta)_{n}$ is one-to-one on $\mathcal{L}_{\nu, r}$.

(b) Let $\omega, \eta, \zeta \in \mathbf{C}$ be chosen as

$$
\begin{aligned}
& \omega=a^{*} \eta+\beta_{1}+\ldots+\beta_{n}-\frac{n+1}{2}-\sum_{i=1}^{n} \alpha_{i} ; \\
& a^{*} \Re(\eta) \geq \gamma(r)-2 a_{1}^{*} \nu+n / 2-\Re\left(\beta_{1}+\ldots+\beta_{n}\right)+\sum_{i=1}^{n} \alpha_{i} ; \\
& \Re(\eta)>-\nu ; \Re(\zeta)<\nu ;
\end{aligned}
$$

where $\gamma(r)$ is given in (2.25). If the condition in (3.5) is satisfied, then

$$
\begin{aligned}
\mathcal{E}(\alpha, \beta)_{n}\left(\mathcal{L}_{\nu, r}\right)=\left(M_{-\frac{1}{2}-\frac{\omega}{2 a_{1}^{*}}} \mathbb{H}_{2 a_{1}^{*}, 2 a_{1}^{*} \zeta+\omega-1} \mathbb{L}_{a^{*}, 1 / 2-\eta+\frac{\omega}{2 a_{1}^{*}}}\right) \\
\left(\mathcal{L}_{\frac{1}{2}-\nu-\frac{\Re(\omega)}{2 a_{1}^{*}}}, r\right) .
\end{aligned}
$$

When the condition in (3.5) is not valid, $\mathcal{E}(\alpha, \beta)_{n}\left(\mathcal{L}_{\nu, r}\right)$ is the subset of the right-hand side of (4.8).

\section{Inversion Formulas}

In this section we present inversion formulas for $\mathcal{E}(\alpha, \beta)_{n^{-}}$transform (1.1) in the case when $a^{*}=2+\sum_{i=p+1}^{n} \alpha_{i}-\sum_{i=1}^{p} \alpha_{i}=0$. Using the inversion for the $\mathbf{H}$ transform $[8,(4.9 .1)$ and (4.9.2)] and taking (2.6) and (3.2)-(3.3) into account, 
we deduce the inversion formulas of $\mathcal{E}(\alpha, \beta)_{n^{-}}$transform in the respective forms:

$$
\begin{aligned}
& f(x)= h x^{1-(\lambda+1) / h} \frac{d}{d x} x^{(\lambda+1) / h} \times \\
& \times \int_{0}^{\infty} H_{n-p+2, p+2}^{p, n-p+1}\left[x t \mid \begin{array}{l}
(-\lambda, h),\left(1-\beta_{i}+\alpha_{i},-\alpha_{i}\right)_{(p+1, n)},(0,1) \\
\left(\beta_{i}-\alpha_{i}, \alpha_{i}\right)_{(1, p)},(0,1),(-\lambda-1, h)
\end{array}\right] \times \\
& \times\left(\mathcal{E}(\alpha, \beta)_{n} f\right)(t) d t
\end{aligned}
$$

or

$$
\begin{aligned}
& f(x)=-h x^{1-(\lambda+1) / h} \frac{d}{d x} x^{(\lambda+1) / h} \times \\
& \times \int_{0}^{\infty} H_{n-p+2, p+2}^{p+1, n-p} {\left[x t \mid \begin{array}{l}
\left(1-\beta_{i}+\alpha_{i},-\alpha_{i}\right)_{(p+1, n)},(0,1),(-\lambda, h) \\
(-\lambda-1, h),\left(\beta_{i}-\alpha_{i}, \alpha_{i}\right)_{(1, p)},(0,1)
\end{array}\right] \times } \\
& \times\left(\mathcal{E}(\alpha, \beta)_{n} f\right)(t) d t
\end{aligned}
$$

The conditions for the validity of the relations (5.1) and (5.2) will be different in the cases when $\sum_{i=1}^{n} \alpha_{i}=0$ and $\sum_{i=1}^{n} \alpha_{i} \neq 0$. The result in the first case follows from [8, Theorems 4.11 and 4.12].

Theorem 10. Let $1<p<n, \alpha_{i}>0(i=1, \ldots, p), \alpha_{i}<0(i=p+1, \ldots, n)$, $\beta_{i} \in \mathbf{C}(i=1, \ldots, n)$ and $\nu \in \mathbf{R}$ be such that $\sum_{i=1}^{p} \alpha_{i}-\sum_{i=p+1}^{n} \alpha_{i}=2$ and let $0<\nu<1,1-\min _{1 \leq i \leq p} \frac{\Re\left(\beta_{i}\right)}{\alpha_{i}}<v<1-\max _{p+1 \leq i \leq n} \frac{\Re\left(\beta_{i}\right)}{\alpha_{i}}$, and $\lambda \in \mathbf{C}, h>0$.

(a) If $\sum_{i=1}^{n} \alpha_{i}(1-\nu)-\sum_{i=1}^{n} \Re\left(\beta_{i}\right)=n / 2$ and if $f \in \mathcal{L}_{\nu, 2}$, then the relation (5.1) holds for $\Re(\lambda)>\nu h-1$, while (5.2) holds for $\Re(\lambda)<\nu h-1$, respectively. (b) If $\sum_{i=1}^{n} \alpha_{i}=0, \Re\left(\beta_{i}\right)=n / 2$ and if $f \in \mathcal{L}_{\nu, r},(1<r<\infty)$, then the relations (5.1) and (5.2) are valid for $\Re(\lambda)>\nu h-1$ and for $\Re(\lambda)<\nu h-1$, respectively.

The conditions for the validity (5.1) and (5.2) in the case when $\sum_{i=1}^{n} \alpha_{i} \neq 0$ follows from [8, Theorems 4.13 and 4.14].

Theorem 11. Let $1<p<n, \alpha_{i}>0(i=1, \ldots, p), \alpha_{i}<0(i=p+1, \ldots, n)$, $\beta_{i} \in \mathbf{C}(i=1, \ldots, n)$ and $\nu \in \mathbf{R}$ be such that $\sum_{i=1}^{p} \alpha_{i}-\sum_{i=p+1}^{n} \alpha_{i}=2$ and let $0<\nu<1,1-\min _{1 \leq i \leq p} \frac{\Re\left(\beta_{i}\right)}{\alpha_{i}}<\nu<1-\max _{p+1 \leq i \leq n} \frac{\Re\left(\beta_{i}\right)}{\alpha_{i}}$, and additionally $-\frac{n+1}{2}<(1-\nu) \sum_{i=1}^{n} \alpha_{i}-\sum_{i=1}^{n} \Re\left(\beta_{i}\right) \leq \frac{1-n}{2}-\gamma(r)$, where $\gamma(r)$ is given in (2.25) and $\lambda \in \mathbf{C}, h>0$. If $f \in \mathcal{L}_{\nu, r},(1<r<\infty)$, then the inversion formulas (5.1) and (5.2) hold for $\Re(\lambda)>\nu h-1$ and for $\Re(\lambda)<\nu h-1$, respectively. 
Remark 1. Taking $p=n$ in Theorems $3,4,5,6,8$ and 11 , we deduce the corresponding result for $\mathcal{E}(\alpha, \beta)_{n}$-transform (1.1) in the case $(\mathbf{A})$ in (2.6) when $\alpha_{i}>0(i=1, \ldots, n)$ and $\mathcal{L}=\mathcal{L}_{-\infty}$.

Remark 2. The result similar to those, given in Section 3-5, stay true for the $\mathcal{E}(\alpha, \beta)_{n}$-transform in the case $(\mathbf{C})$ when $1<p<n, \alpha_{i}<0(i=1, \ldots, p)$, $\alpha_{i}>0(i=p+1, \ldots, n)$ and either $\sum_{i=1}^{n} \alpha_{i}>0, \mathcal{L}=\mathcal{L}_{-\infty}$ or $\sum_{i=1}^{n} \alpha_{i}<0$, $\mathcal{L}=\mathcal{L}_{+\infty}$. Taking $p=n$, the corresponding result can be deduced for the $\mathcal{E}(\alpha, \beta)_{n}$-transform (1.1) in the case $(\mathbf{D})$ in $(2.6)$ when $\alpha_{i}<0(i=1, \ldots, n)$ and $\mathcal{L}=\mathcal{L}_{+\infty}$. See Remark 1 in this connection.

\section{Acknowledgements}

The present investigation was partly supported by the Belarusian Fundamental Research Fund (Project F05MC-050).

\section{References}

[1] M.M. Dzhrbashyan. On integral transforms generated by generalized MittagLeffler function. Izv. Akad. Nauk Armyan. SSR, Ser. Fiz.-Mat., 13(3), $21-63$, 1960. (In Russian)

[2] M.M. Dzhrbashyan. Integral Transforms and Representations of Functions in the Complex Domain. Nauka, Moscow, 1968. (Russian)

[3] M.M. Dzhrbashyan. Harmonic Analysis and Boundary Value Problems in the Complex Domain. Operator Theory: Advances and Applications, volume 65. Birkhauser Verlag, Basel, 1993.

[4] A. Erdelyi, W. Magnus, F. Oberhettinger and F.G. Tricomi. Higher Transcendental functions, volume 2. McGraw-Hill, New York-Toronto-London, 1953. Reprinted Krieger, Melbourne, Florida, 1981

[5] A. Erdelyi, W. Magnus, F. Oberhettinger and F.G. Tricomi. Higher Transcendental Functions, volume 3. McGraw-Hill, New York-Toronto-London, 1955. Reprinted Krieger, Melbourne, Florida, 1981

[6] S.B. Hadid and Y.F. Luchko. An operational metthod for solving fractional differential equation of an arbitrary real order. Panamer. Math. J., 6(1), 57$73,1996$.

[7] A.A. Kilbas and A.A. Koroleva. Generalised Mittag-Leffler function and its extension. Tr. Inst. Mat. Minsk, 13(1), 43-52, 2005. (In Russian)

[8] A.A. Kilbas and M. Saigo. H-Transform. Theory and Applications. Chapman and Hall/CRC, Boca Raton-London-New York-Washington, D.C., 2004.

[9] A.A. Kilbas and J.J. Trujilo. Differential equations of fractional order: methods, results and problems. Appl. Anal., 78(1-2), 153-192, 2001.

[10] A.M. Mathai and R.K. Saxena. The H-Function with Applications in Statistics and other Disciplines. Halsted Press, New York-London-Sydney, 1978.

[11] A.P. Prudnikov, Yu.A. Brychkov and O.I. Marichev. Integrals and Series, volume 3. More Special Functions. Gordon and Breach, New York, etc., 1990.

[12] S.G. Samko, A.A Kilbas and O.I. Marichev. Fractional Integrals and Derivatives. Theory and Applications. Gordon and Breach, Yverdon, 1993.

[13] H.M. Srivastava, K.C Gupta and S.L. Goyal. The H-Function of One and Two Variables with Applications, volume 6. South Asian Publishers, New-DelhiMadras, 1982. 